\title{
APPLICATION OF DSM IN OBSTACLE CLEARANCE SURVEYING OF AERODROME
}

\author{
X. Qiao ${ }^{\text {a, }}$, S. H. Lva, b, L. L. Li ${ }^{\text {c }}$, X. J. Zhou ${ }^{\text {a }}$, H. Y. Wang a , D. Li ${ }^{\text {a }}$ J. Y. Liu ${ }^{\text {a }}$ \\ ${ }^{a}$ Qingdao Geotechnical Investigation and Surveying Institute, State and Local Joint Engineering Research Center for the \\ Integration and Applicatioin of Sea-land Geographical Information, Shandong Road, Qingdao, China - (49984066@qq.com, \\ 1shmm2001@aliyun.com, 529130998@qq.com, why@qdkcy.com.cn, ld_sd@163.com, kcyjcb@163.com) \\ ${ }^{\mathrm{b}}$ College of Environmental Science and Engineering, Ocean University of China, Songling Road, Qingdao, China - \\ 1shmm2001@aliyun.edu.cn \\ ${ }^{\mathrm{c}}$ College of Information Science and Engineering, Ocean University of China, Songling Road, Qingdao, China - lilele@ ouc.edu.cn
}

\section{Commission II, WG II/3}

KEY WORDS: Obstacle Clearance Surveying, Obstacle Limitation Surface, Digital Surface Model, Spatial Analysis

\begin{abstract}
:
Compared to the wide use of digital elevation model (DEM), digital surface model (DSM) receives less attention because that it is composed by not only terrain surface, but also vegetations and man-made objects which are usually regarded as useless information. Nevertheless, these objects are useful for the identification of obstacles around an aerodrome. The primary objective of the study was to determine the applicability of DSM in obstacle clearance surveying of aerodrome. According to the requirements of obst acle clearance surveying at QT airport, aerial and satellite imagery were used to generate DSM, by means of photogrammetry, which was spatially analyzed with the hypothetical 3D obstacle limitation surfaces (OLS) to identify the potential obstacles. Field surveying was then carried out to retrieve the accurate horizontal position and height of the obstacles. The results proved that the application of DSM could make considerable improvement in the efficiency of obstacle clearance surveying of aerodrome.
\end{abstract}

\section{INTRODUCTION}

\subsection{Obstacle Identification}

In order to permit the intended airplane operations at the aerodromes to be conducted safely and to prevent the aerodromes from becoming unusable by the growth of obstacles around the aerodromes, ICAO provides a series of standards of obstacle limitation surfaces (OLS) that define the limits to which objects may project into the airspace (ICAO, 2009).

The OLS of an aerodrome consists of a series of hypothetical surfaces: conical surface, inner horizontal surface, approach surface, inner approach surface, transitional surface, inner transitional surface, balked landing surface, take-off climb surface, as shown in Figure 1. These surfaces, which are formed according to different mathematical models, have different dimensions and slopes that are provided in <Aerodrome Design Manual> (ICAO Doc 9157) and <Aerodrome Technical Standards (MH 5001-2013)>, the Chinese version of ICAO Doc 9157. When one OLS overlaps with another surface, the obstacle at any point inside the overlap area must be controlled with respect to the lowest OLS. In this sense, the whole OLS is constructed of the lowest limitation surface at any point.

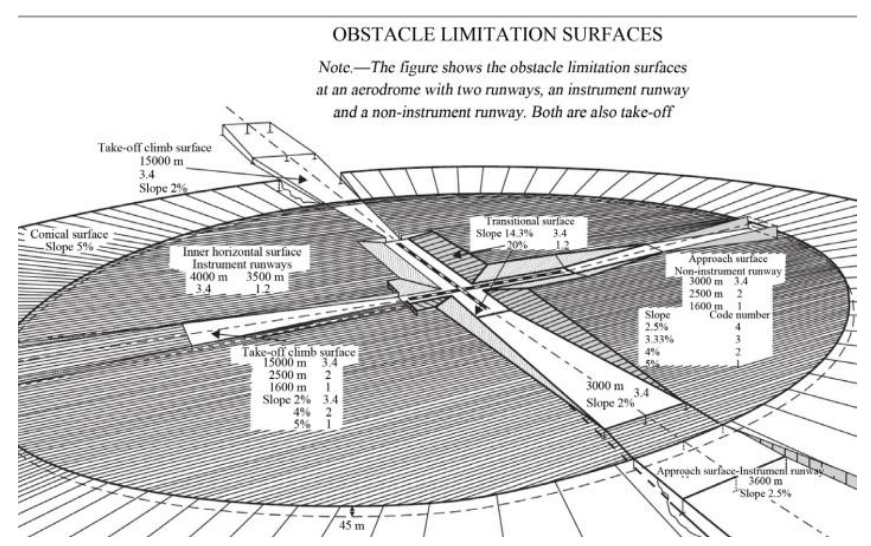

Figure 1. Obstacle limitation surfaces, Annex 14, Att. B

Any object that penetrates these surfaces is identified as an obstacle and should be surveyed to retrieve the accurate horizontal position and height, then be demolished or removed to ensure the safety of airplane landing, climbing or circling.

The obstacles are divided into two types: natural obstacles and man-made obstacles. The natural obstacles include vegetations and terrains above the OLS, while the man-made obstacles include objects above the OLS such as poles, towers, chimneys, buildings etc. Additionally, a height of objects such as train, vehicle, and ship, should be added to the terrain elevation within areas of railways, roads and rivers.

\footnotetext{
* Corresponding author
} 
The conventional method of obstacle clearance surveying is to retrieve the spatial distribution of potential obstacles by comparing the complex OLS with the objects elevation extracted from two-dimensional topographic maps or investigation results with the local government, and then to carry out a field surveying of the potential obstacles using GPS or total station to achieve the accurate height used to identify the real obstacles (Wang et al., 2012; Niu et al., 2000; Fu et al., 2005).

Due to the difficulty of spatially analyzing elevation from 2D topographic maps with complex 3D OLS, especially on condition of multi-runways, it's hard to analyze the accurate spatial relationships between the potential obstacles and the OLS correctly and efficiently. Moreover, the large amounts of investigation, data acquisition and fieldwork in large areas also contribute for the high cost of obstacle identification, especially in areas short of fundamental geographic data and of complex terrain conditions.

To solve these problems, studies were focused on the 3D visualization of OLS (Li et al., 2011; Ma et al., 2008; Wang et al., 1998; Yi et al., 2010) and its application on obstacle identification (Fang et al., 2010; Cai et al., 2004; Yang et al., 2012; Luo et al., 2011; Li, 2011). Scholars constructed 3D model of OLS using AutoCAD, 3DsMax or ArcGIS, and then identified obstacles by comparing the OLS model with the objects height retrieved from topographic maps, field surveying or DEM.

The disadvantage of previous studies is that the data used to be compared with OLS is either costly in time and money to acquire or not a true expression of the world. For example, the DEM generation is time-consuming and costly in filtering none-ground objects like buildings and vegetations, usually in a manual method, while these objects are main sources of obstacles. On the other hand, topographic maps used to extract the height usually got a relatively not good temporal property, which would lead to the missing of newly changed objects.

\subsection{Digital Surface Model}

DSM represents the earth's surface and includes all objects. The elevation values refer to the upper vegetation canopy or to the roof of man-made objects. In contrast to digital terrain model (DTM) or DEM, DSM supplies more spatial information of above ground features, thus gained much attention among environment scientists on ortho-image generation, building extraction and change detection in urban areas, forest variables derivation and deforestation monitoring, hydrological analysis, geomorphology monitoring and estimation of city climate conditions etc.

Using aerial image stereo pairs, Garouani constructed the DSM of Fez urban communities and applied it in the 3D city planning by extracting building volumes, usage and density from DSM (Garouani et al., 2014). Liu introduced a method to detect man-made objects change by means of height change, line-feature matching and gradient direction histogram from comparison between new and old DSMs (Liu et al., 2003). Gomez demonstrated the potentials to extract topographical and vegetation data, and the potentials for chronological analysis of landscape evolution using structure from motion derived DSMs and DEMs based on historical aerial photographs (Gomez et al., 2015). Nurminen compared the performance of DSM extraction using photogrammetric images matching and laser scanning in the estimation of plot-level forest variables (Nurminen et al., 2013). Avtar used PRISMDSM, SRTM-DEM and ASTER-GDEM data to monitor changes in height of forests caused by deforestation activity in Cambodia (Avtar et al., 2013). Nikolakopoulos used DSM from ALOS, CARTOSAT and aerophotographs stereo-pairs to extract the contour, the drainage network, the hydrological basin and the calculation of three hydrological indexes (Nikolakopoulos et al., 2011). Bogdanović introduced how DSM derived from LIDAR technology can contribute qualitatively and quantitatively for research and monitoring of rock slopes (Bogdanović et al., 2015). Johansson attempted to model the relationship between near-ground wind speed at street level and urban geometry represented by high resolution DSM (Johansson et al., 2015).

As mentioned in section 1.1, 3D visualization of OLS and identification of obstacles using spatial analysis of OLS and DEM were focused by scholars recently. While there are few studies concentrated on the subject of using DSM for obstacle identification. Zhao analyzed the relationship between the spatial intervals of DSM and the isolated obstacle detection ability (Zhao et al., 2003). The simulation results showed that the vertical and horizontal intervals between $3 \mathrm{~m}$ and $25 \mathrm{~m}$ could reach the balance of accuracy and time-cost to accurately identify isolated obstacles for aircraft precision terrain following guidance.

The primary objective of the study was to develop an economical and accurate method for identifying obstacles by using DSM and 3D GIS technology.

\section{MATERIAL}

\subsection{Study Area}

As to the OLS design is beyond the scope of this study, we directly used the "QT airport obstacle limitation surface map" which presents the OLS in a 2D style as shown in Figure 2. As presented in the design map, there are two areas where obstacles need to be identified:

Area 1: areas within the conical surface (including), which is about 320 square kilometers.

Area 2: the outer horizontal surface, which is a plane located $150 \mathrm{~m}$ above the aerodrome elevation datum and extending from the upper edge of the extended conical surface for a distance of 50,000 $\mathrm{m}$ (radius) from aerodrome reference point (ARP). Area 2 is about 7500 square kilometers.

The obstacle limitation criteria of the two OLS areas are different. Within area 1 , any object violates the obstacle limitation criteria as mentioned in section 1.1 is regarded as an obstacle, while only terrain that is above the outer horizontal surface is regarded as an obstacle within area 2. 


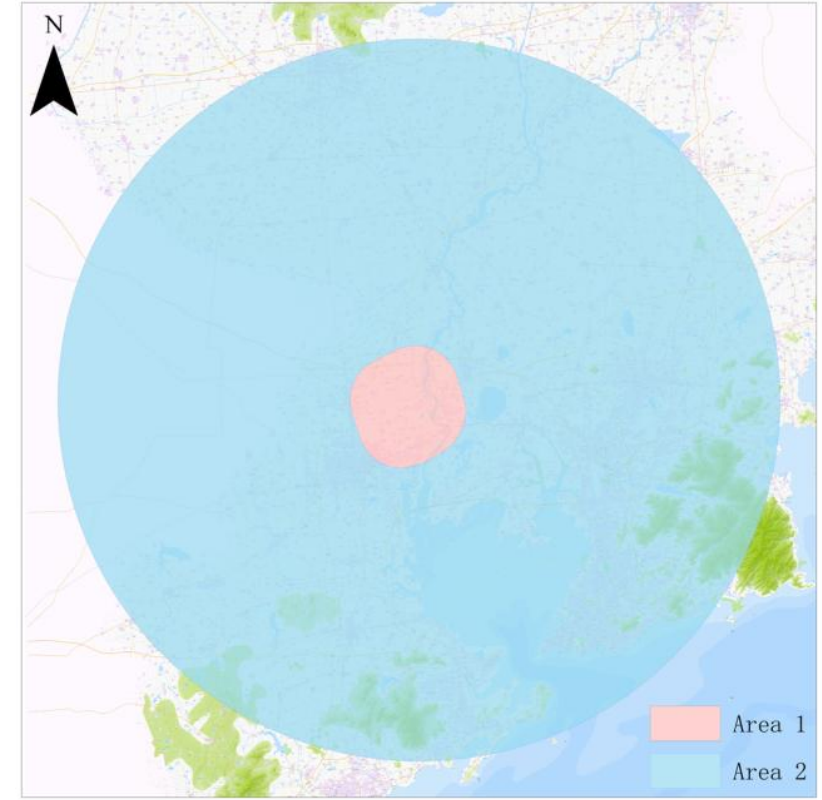

Figure 2. Obstacle limitation surface areas of QT airport

\subsection{Digital Aerial and Satellite Imagery}

Generally speaking, site of new airport is selected in rural areas, where fundamental geographic data like topographic map or DOM is rare. Technical route for data acquisition need to be discussed carefully to ensure the accuracy within limited budget. At present, main methods of generating DSM include laser intensity direction and ranging (LIDAR), photogrammetry using aerial or satellite images with stereo coverage. LIDAR was abandoned due to the low flight efficiency, which results from a slow flight speed needed to retrieve very close point density.

For the large area of the overall obstacle identification area, which is about 7800 square kilometers, aerial imagery acquisition is not an economical and efficient way to retrieve image data. On the other hand, obstacle limitation criteria are different in two areas. So, two ways of image acquisition were adopted in this study on the principles of economy and efficiency: aerial imagery was acquired by means of the Airborne Digital Sensor 100 (ADS100) within area 1, and ZY3 satellite images were used within area 2 .

The flights within area 1 were undertaken with a ground sample distance (GSD) of $0.05 \mathrm{~m}$. The ADS100 is part of the first commercially available digital large format airborne survey systems introduced in 2013. The ADS systems' basic design is the three-linescanner principle, whereby linear arrays on the focal plane capture imagery looking forwards, downwards (nadir) and backwards from the aircraft. The ground surface is imaged multiple times within one flight line, which means it could provide stereo overlap of up to $90 \%$ or entire image strips with higher radiometric resolution. The ADS sensor systems capture imagery by a total of 13 CCD lines of red, green, blue and near infrared data. Each line consists of 20,000 pixels with a size of $5 \mu \mathrm{m}$.

As the nation's first high-resolution optical transmission stereo satellite, ZY-3 was launched on January 9 2012, and be delivered on July 30 formally for full operation. The satellite is equipped with three panchromatic cameras for front, direct and back view vision to capture omni-direction images with $100 \%$ coverage. ZY-3 features panchromatic resolution finer than 2.1 $\mathrm{m}$ and multi-spectral resolution finer than $6 \mathrm{~m}$. ZY-3 is the first Chinese remote sensing satellite capable of stereo imaging from different angles in multi-spectrum. It integrates the functions of surveying and mapping with resource investigation together and is mainly used for 1:50000 scale stereo mapping and digital imaging as well as the updating of some elements in the 1:25000 scale topographic maps. Since delivery, ZY-3 played an important role in surveying and mapping of West China for further exploration, obtaining geoinformation of coastal line and island environment to better protect the country's ocean rights, and providing stable satellite remote sensing data for urban planning, agriculture, forestry, irrigation and biological environment monitoring.

For the overall study area, 31 flight lines were carried out within area 1 as shown in Figure 3, and 8 scenes of satellite images were adopted as shown in Figure 4.

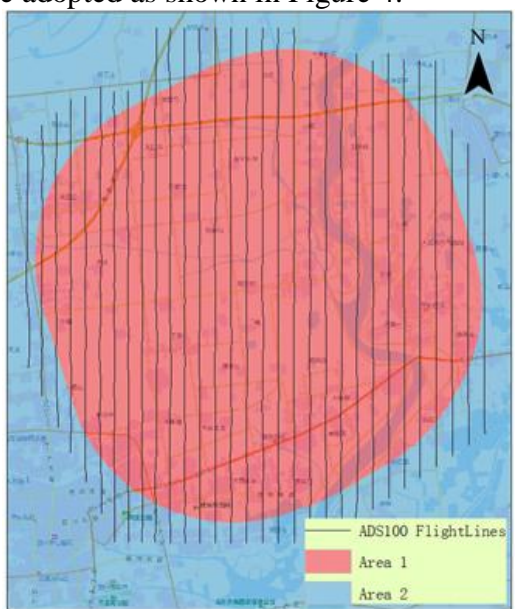

Figure 3. ADS100 flight lines

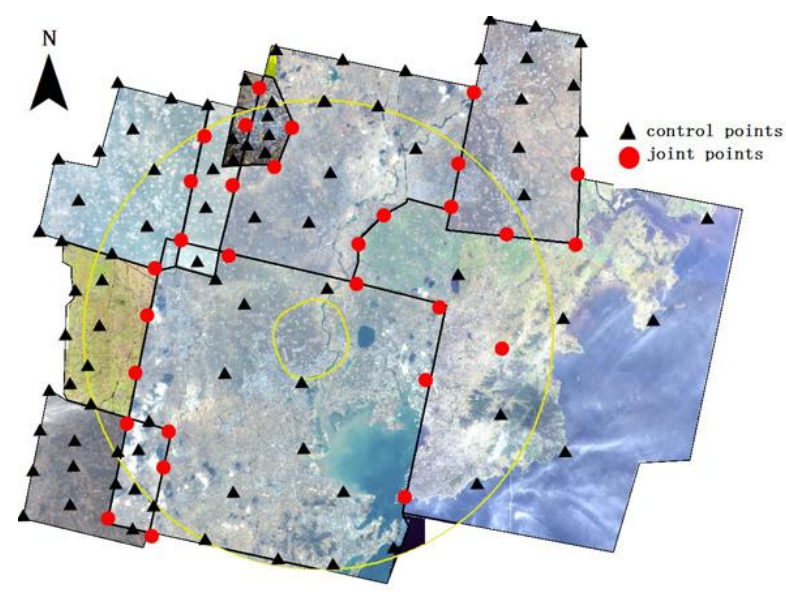

Figure 4. ZY-3 Scenes and control points

\section{METHOD}

\subsection{Workflow}

Workflow of the study is shown in Figure 5. 


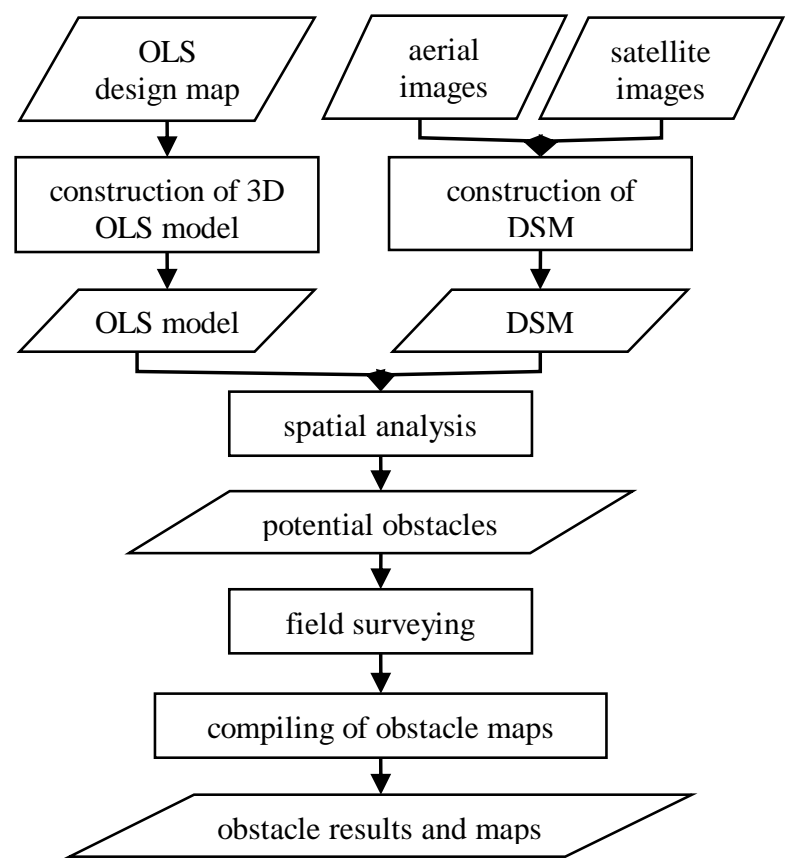

Figure 5. Workflow of the study

\subsection{D Construction of OLS Model}

3D OLS model could not only be used in the identification of obstacles before aerodrome construction, but also play an important role in the subsequent safety management for aerodrome maintenance (Mao et al., 2010). Software like AutoCAD, 3dsMax, ArcGIS is adopted in 3D construction of objects usually. In this study we adopted ArcGIS 10.2 to rebuild the 3D OLS model.

The OLS design map is composed by 2D contours which represent the slope and dimension of every limitation surface in DWG format. Only the contours of the lowest surface remained in area where several surfaces overlap. In this study, all surfaces were processed separately and be assembled to a complete surface model.

Contours of every limitation surface were converted to shape file format in ArcGIS, and then be generated to triangulated irregular network (TIN) which was constructed by triangulating a set of vector-based vertices. These vertices were connected with a series of edges to form a network of triangles which was formed by means of the Delaunay triangulation method of interpolation.

TIN is the most accurate 3D surface model compared to the other three types of surface models: raster, terrain dataset, and LAS dataset for the fact that the input features used to generate a TIN model remain in the same position as the nodes or edges in the TIN, which allows a TIN model to preserve all the precision of the input data while simultaneously modelling the values between known points. However, TIN model is less widely available than raster surface models, because it tends to take longer to build and require much more disk space due to the complex data structure (Kennedy, 2009). As a result, in order to be analyzed with raster-based DSM more efficiently, TINs were converted to raster format by means of interpolation at a specified sampling distance, which is $1 \mathrm{~m}$ in this study to reach a balance between efficiency and accuracy.

After the conversion of 3D raster surfaces from OLS TIN models, assembly of these separate raster surfaces was implemented to form a single raster surface using a mosaic method supplied within ArcGIS. For overlap area, the lowest pixel value is adopted by means of "minimum" method.

It couldn't be forgotten that height of these surfaces in design is relative to the elevation of the highest point of the landing area i.e. aerodrome elevation. So, the complete OLS model was moved in vertical direction with an offset of the aerodrome elevation.

\subsection{Generation of Digital Surface Model}

Spatial accuracy of obstacles in this study was supposed to be consistent with the corresponding provisions in <Aerodrome technical Standards> (MH 5001-2013) as shown in Table 1. As described in this table, accuracy requirements are different within area 1 and area 2 . Therefore, in order to meet the higher accuracy requirement, DSM generation within area 1 was carried out by a stringent method, i.e. aerial imagery acquisition with a GSD of $0.05 \mathrm{~m}$. As to the DSM generation within area 2, satellite images which could meet the accuracy requirements with a GSD of less than $1 \mathrm{~m}$, was selected to reach a balance between efficiency and cost.

\begin{tabular}{|c|c|}
\hline Elevation & Accuracy \\
\hline Area 1 & $0.5 \mathrm{~m}$ \\
Area 2 & $3.0 \mathrm{~m}$ \\
\hline
\end{tabular}

Table 1. Accuracy of obstacle surveying

For the generation of DSM within area 1, we used Leica XPro and VirtuoZo. The former was used in aerial triangulation, while the latter was used to generate DSM based on the aerial triangulation result. In order to control the accuracy as high as possible, five ground control points were surveyed and participated in the aerial triangulation combined with IMU/DGPS data in XPro. XPro is a software package, licensed by Leica Geosystems, and performs the ADS ground processing workflow from data download to image generation and dense DSM extraction.VirtuoZo was initially proposed by Prof. Wang as a prototype called Wuhan Digital Automatic Mapping System (WuDAMS), and was developed to a commercial photogrammetric software later (Zhang et al., 1996).

Followed the aerial triangulation, exterior orientation parameters of images were transferred to be compatible with ADS module of VirtuoZo for generation of DSM. Image matching was then performed to derive a regularly spaced cloud of point, i.e. the DSM. In order to identify as little as possible potential obstacles within area 1, the grid spacing of DSM was set to $0.1 \mathrm{~m}$, although this led to huge amount of data. On the other hand, the grid spacing is depended on the terrain type. For example, relative coarse grid spacing could meet the requirements in flat terrain, while fine grid spacing is needed to represent the complex terrain accurately. Finally, the point cloud within area 1 was converted to a raster-based DSM of ASCII format.

As to the satellite image processing within area 2, VirtuoZoSAT is used. As a satellite image processing module, VirtuoZoSAT could process many satellite data sources such as 
IKONOS/GeoEye-1, QuickBird, WorldView, SPOT5, OrbView, P5, ALOS PRISM, EROS, GF-1, etc. DSM generation using satellite imagery is similar to that of aerial imagery. Firstly, images on the same track were rotated 90 degrees in order to delineate the vertical parallax before import into a project. Next, 107 ground control points were imported into the project and tie points were extracted automatically to finish absolute triangulation, which followed by image matching to generate DSM.

\subsection{Obstacle Identification}

Prior to the spatial analysis of DSM and OLS model, as described in section 1.1, the height of a train/vehicle/ship was supposed to be added to the DSM in areas of railways, roads, and rivers. This was a relatively complex work including digital orthophoto map (DOM) generation, railway/road/river vectorization and conversion to raster surfaces, and addition to the DSM. Using DSM data, we rectified the images and mosaicked to DOM, which was used as the basic data to draw the outlines of railways, roads and rivers in ArcGIS. Next, a certain height (e.g., $7.5 \mathrm{~m}$ for train, $10 \mathrm{~m}$ for vehicle) was added to different areas in the raster model converted from vector-based outlines. Finally, these raster models were merged to previous DSM data.

After the OLS and DSM were ready, minus operation was carried out to subtract the value of the OLS from the value of the DSM on a cell-by-cell basis to get the DSM pixel above the OLS, i.e. the potential natural and man-made obstacles.

\subsection{Field Surveying of Obstacles}

Field surveying of obstacles was implemented according to the potential obstacle distribution achieved from the above works. In this study, we set up mapping control points around the areas of the potential obstacles using continuous operational referencing system (CORS), and then made a field surveying of the potential obstacles using total station by means of polar coordinates method. It should be noted that the nearest horizontal position to the ARP and the highest point of the obstacles such as lightning rod, antenna on buildings, were to be surveyed. Additionally, number and field photo of obstacles were registered on the spot.

\subsection{Compiling of obstacle results and maps}

Prior to the achievement of final results, the height of potential obstacles retrieved from field surveying was compared with the OLS model to determine whether these objects really penetrated OLS.

Then, obstacle topographic map and information table were compiled as the final results of obstacle clearance surveying. As shown in Figure 6, obstacle topographic map, usually in a scale of 1:50000, was composed by the runways and obstacles expressed by symbols for manmade objects and contours for terrains. Moreover, number and height were labelled beside the obstacle symbol. In this study, there was no obstacles in area 2. A fraction of the obstacles information was shown in Table 2 .

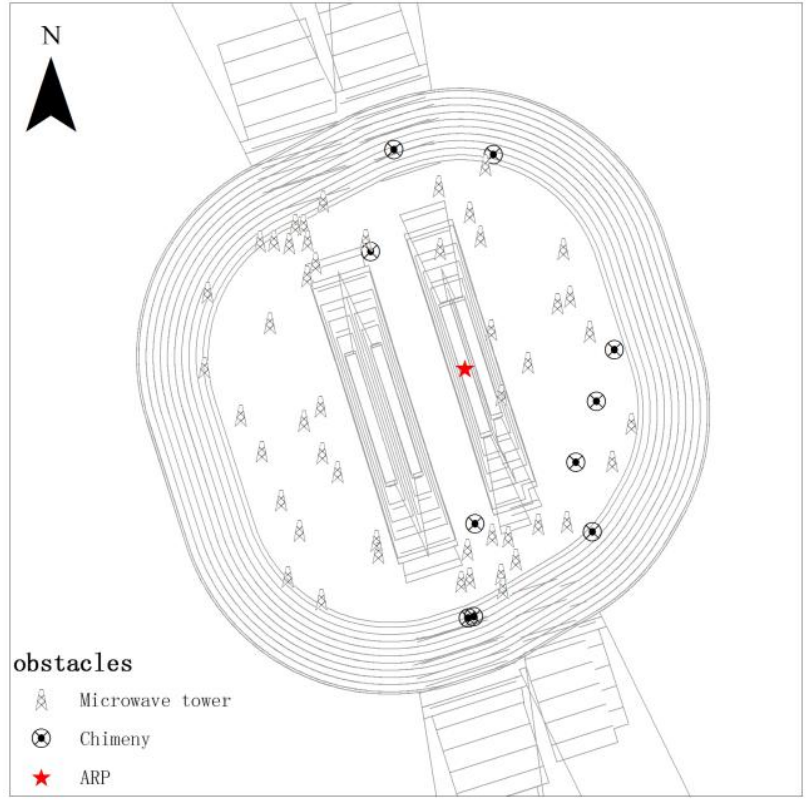

Figure 6. Obstacle topographic map

\begin{tabular}{|c|c|c|c|}
\hline \multirow{2}{*}{ obstacle name } & \multicolumn{2}{|c|}{ WGS-84 coordinates } & \multirow{2}{*}{$\begin{array}{c}\text { elevation } \\
\text { (m) }\end{array}$} \\
\hline & longitude & latitude & \\
\hline $\begin{array}{c}\text { Microwave } \\
\text { tower A }\end{array}$ & $\mathrm{E}^{* * \circ} 09^{\prime} 03.9^{\prime \prime}$ & $\mathrm{N}^{* * \circ} 21^{\prime} 06.8 "$ & $* * .418$ \\
\hline $\begin{array}{c}\text { Microwave } \\
\text { tower B }\end{array}$ & $\mathrm{E}^{* * \circ} 055^{\prime} 52.5^{\prime \prime}$ & $\mathrm{N}^{* * 0} 18 \mathrm{8} 43.6 "$ & $* * .844$ \\
\hline Chimney A & $\mathrm{E}^{* * \circ} 02 ' 51.4 "$ & $\mathrm{~N}^{* * \circ} 19 ' 30.1 "$ & $* * .46$ \\
\hline
\end{tabular}

Table 2. Obstacle information of aerodrome

\section{CONCLUSION}

Followed the above steps, we identified the obstacles within OLS areas of QT airport by means of spatial analysis of OLS model and DSM in a 3D environment, and mad a field surveying to retrieve the horizontal position and height which were used to determine the real obstacles. The total duration was 35 days which decreased about $50 \%$ compared to the conventional methods of obstacle identification.

The results indicate that the identification of obstacles by means of spatial analysis of 3D OLS model and DSM has great potential as a cost-effective method. The advantages of this method are high accuracy and time-cost-efficient, all steps automatic compared to the conventional method. Nevertheless, much investment is needed to carry out the data acquisition, though the derivation of DSM could serve as important fundamental data in the future safety management of the airport, such as 3D simulation flight, earthwork calculation, and identification of newly built objects or extensions.

In future studies, further works are supposed to be directed towards the DSM generation using higher-resolution satellite imagery. Compared to the aerial imagery acquisition and processing, satellite imagery photogrammetry has many advantages: low cost in data acquisition, all-weather data acquisition, bigger coverage and simpler data processing. 
Along with the increasing of GSD, satellite imagery is bound to replace the aerial imagery in more areas. For example, launched on August 13 2014, WorldView III with a GSD of $0.31 \mathrm{~m}$ could be applied in the generation of topographic map in a scale of 1:3000, while which was generated only by using aerial imagery previously.

\section{REFERENCES}

Aerodrome technical standards (MH 5001-2013). CAAC, 2013.

Avtar R., Sawada H., 2013. Use of DEM data to monitor height changes due to deforestation. Arabian Journal of Geosciences, 6, pp. 4859-4871.

Bogdanović S., Marjanović M., Abolmasov B., Basarić U. I., 2015. Rockfall monitoring based on surface models. Surface Models for Geosciences, 2015, pp. 37-44.

Cai X. Q., Miao T. B., Cao J. J., Duan X., Mo J. K., Tong J. Y., 2004. Three-dimensional analysis of obstacle in the limited plane of Qingyang airport clearance. Remote Sensing Technology and Application, 19(5), pp. 407-410.

Fang X. D., Liu X. P., 2010. Study on the conical surface obstacle encroaching evaluation of civil aerodrome. Aeronautical Computing Technique, 40(1), pp. 46-49.

Fu Z. A., Bao H., Peng X. G., Wang Y. F., 2005. The measurement of the airfield clearance area obstacle using GPS/GSM and total station. Bulletin of Surveying and Mapping, 2005(3), pp. 38-39, 45.

Garouani A. E., Alobeid A., Garouani S., 2014. Digital surface model based on aerial image stereo pairs for 3D building. International Journal of Sustainable Built Environment, 3(1), pp. 119-126.

Gomez C., Hayakawa Y., Obanawa H., 2015. A study of Japanese landscapes using structure from motion derived DSMs and DEMs based on historical aerial photographs: New opportunities for vegetation monitoring and diachronic geomorphology. Geomorphology in the Geocomputing Landscape: GIS, DEMs, Spatial Analysis and stastics, 242, pp. 11-20.

ICAO (2009) Aerodrome Design and Operations. Annex 14, Vol. 1, 5th Edition, ICAO, Montreal.

ICAO (2006) Aerodrome Design Manual. Doc 9157, 3rd Edition, Montreal.

Johansson L., Onomura S., Lindberg F., Seaquist J., 2015. Towards the modelling of pedestrian wind speed using highresolution digital surface models and statistical methods. Theoretical and Applied Climatology, 2015: 1-15

Kennedy H., 2009. Introduction to 3D Data: Modeling with ArcGIS 3D Analyst and Google Earth. John Wiley \& Sons, Inc.

Li J., 2011. 3D Visualization Techniques Research of Aerodrome Clearance Obstacle Assessment. Dissertation for the Degree of Master.
Li P., Cai L. C., Liu Z., Li B., 2011. Building the mathematical model of airfield obstacle limitation surfaces and visualizing the model. Science of Surveying and Mapping, 36(2), pp. 198200.

Liu H., 2012. ZY-3 High Resolution Satellite Delivered for Operation. Aerospace China, 13(3), pp. 17.

Liu Z. F., Zhang J. Q., Zhang Z. X., Fan H., 2003. Change detection based on DSM and image features in urban areas. Geo-spatial Information Science. 6(3), pp. 35-41.

Luo C. L., Cai L. C., Zhang L. L., Meng D. S., 2011. Obstacle assessment of parallel double-runway airfield clearance. Journal of Traffic and Transportation Engineering, 11(1), pp. $69-73,88$.

Ma Y. F., Wang W. A., 2008. Research on the computing model of airport clearance limitation and its visualization. Engineering of Surveying and Mapping, 37(1), pp. 32-36.

Mao W. Q., Wang W. A., 2010. GIS applied in airfield clearance management. Science of Surveying and Mapping, 35(4), pp. 195-197.

Nikolakopoulos K., Gioti E., 2011. Suitability of DSM derived from remote sensing data for hydrological analysis with reference to the topographic maps of 1/50000. Advances in the Research of Aquatic Environment, pp. 121-128.

Niu Z. B., Zhang C. A., 2000. Three-dimensional survey of obstacle points in the limited plane of airport clearance. Northwest surveying and mapping, 23(1), pp. 22-23.

Nurminen K., Karjalainen M., Yu X. W., Hyyppa J., Honkavaara E., 2013. Performance of dense digital surface models based on image matching in the estimation of plot-level forest variables. ISPRS Journal of Photogrammetry and Remote Sensing, 83, pp. 104-115.

Wang J. G., Wang C. L., Huang S. Q., Shi Y. H., 2012. Application of triangulation in obstruction survey and precision analysis. Science of Surveying and Mapping, 37(2), pp. 179181.

Wang Y. L., Su T. Y., 1998. Drawing of airport clearance limit and obstacle location maps. Engineering of Surveying and Mapping. 7(2), pp. 48-52.

Yang L.F., Shen M. W., Zong Q., 2012. Airborne LiDAR technology and aerial remote sensing images in the survey of the airport clearance protection area obstruction. Urban Geotechnical Investigation \& Surveying. 2012(4), pp. 80-82, 85.

Yi W., Xu J. K., 2010. The research and development of GISbased 3D obstacle-free-space management system for multirunway aerodrome. Science of Surveying and Mapping, 35(1), pp. 177-178, 145.

Zhang J. Q., Zhang Z. X., Shen W. W., Wang Z. H., 1996. VirtuoZo Digital Photogrammetry System and Its Theoretical Foundation and Key Algorithm. In: International Archives of 
Photogrammetry and Remote Sensing, Vienna, Austria, Vol. XXXI, Part B2, pp. 238-245.

Zhao M., Lin X. G., Zhao N. G., Wang Q., 2003. Flight obstacle scaling in a digital surface model. Journal of Tsinghua University (Science \& Technology), 43(9), pp. 12491252.

Revised April 2016 